\title{
GSK-3ß-mediated fatty acid synthesis enhances epithelial to mesenchymal transition of TLR4-activated colorectal cancer cells through regulation of TAp63
}

\author{
GA BIN PARK $^{1 *}$, YOON HEE CHUNG ${ }^{2 *}$, JI HEE GONG ${ }^{3}$, DONG-HOON JIN ${ }^{3,4}$ and DAEJIN KIM ${ }^{5}$ \\ ${ }^{1}$ Department of Biochemistry, Kosin University College of Medicine, Busan 49267; ${ }^{2}$ Department of Anatomy, \\ Chung-Ang University College of Medicine, Seoul 06974; Departments of ${ }^{3}$ Convergence Medicine, and ${ }^{4}$ Oncology, \\ University of Ulsan College of Medicine, Asan Medical Center, Seoul 05505; ${ }^{5}$ Department of Anatomy, \\ Inje University College of Medicine, Busan 47392, Republic of Korea
}

Received June 21, 2016; Accepted August 23, 2016

DOI: 10.3892/ijo.2016.3679

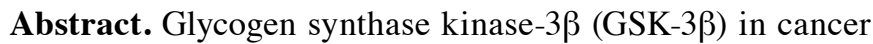
cells is a critical regulatory component of both cellular metabolism and epithelial-mesenchymal transition (EMT) processes via regulation of the $\beta$-catenin/E-cadherin and phosphoinositide 3-kinase (PI3K)/AKT signaling pathway. Lipogenesis of cancer cells also plays a critical role in survival and metastasis. We investigated the role of GSK-3 $\beta$-mediated intracellular fatty acid synthesis to control EMT in TLR4activated colorectal cancer cells and the underlying regulatory mechanism. Engagement of TLR4 with lipopolysaccharide (LPS) in colon cancer cells promoted the induction of phosphorylated GSK-3 $\beta$ and related lipogenic enzymes as well as the expression of CD74, CD44 and macrophage inhibitory factor (MIF), but decreased expression of transcriptionally active p63 (TAp63). In addition, targeted inhibition of GSK-3 $\beta$ using SB216763 was accompanied by decreased intracellular fatty acid synthesis and blockage of CD74 and CD44 expression, whereas it reversed the level of TAp63. Although TAp63 overexpression had no effect on the expression of CD74 and CD44 in LPS-treated colon cancer cells, GSK-3 $\beta$-dependent fatty acid synthesis and invasive activity were significantly suppressed. Notably, inhibition of CD44 or CD74 by siRNA not only attenuated de novo lipogenesis and migratory activity but also restored the expression of TAp63 in LPS-activated colon cancer cells. These results suggest that TAp63-mediated GSK-3 $\beta$ activation induced by TLR4 stimulation triggers
\end{abstract}

Correspondence to: Professor Daejin Kim, Department of Anatomy, Inje University College of Medicine, 75, Bokji-ro, Jin-gu, Busan 47392, Republic of Korea

E-mail: kimdj@inje.ac.kr

*Contributed equally

Key words: GSK-3 $\beta$, TAp63, TLR4, lipogenesis, epithelial mesenchymal transition, colorectal cancer migration and invasion of colon cancer cells through the regulation of lipid synthesis and GSK-3 $\beta$-mediated CD74/CD44 expression could be a target to control fatty acid-related EMT process through the modulation of TAp63 expression.

\section{Introduction}

GSK-3 $\beta$ is believed to play an important regulatory role in cell metabolism, carcinogenesis and targeted therapy in cancer cells (1). GSK-3 $\beta$ is expressed ubiquitously, regulated by diverse stimuli and involved in various signaling pathways, including antitumor protein p53 (TP53) activity $(2,3)$. TP53 regulates Snail expression, which is also regulated by GSK-3 $\beta$. Epithelial-mesenchymal transition (EMT) is promoted through the activation of Snail protein expression after loss of TP53 activity (4). TP53 is normally a suppressor of $\mathrm{Wnt} / \beta$-catenin signaling, which is associated with activation of intracellular $\beta$-catenin regulated by GSK-3, resulting in loss of E-cadherin and mesenchymal phenotype (5-7). Expression of p63, a member of the p53 family, is regulated by the TP63 gene (8), which is expressed as multiple isoforms with distinct properties. The transcriptionally active p63 (TAp63) isoform contains a transcription domain and can induce cell cycle arrest and apoptosis (9); however, the N-terminal-deleted p63 isoform $(\Delta \mathrm{Np} 63)$ has distinct functions, including induction of stem cell characteristics, progression of the cell cycle and enhancement of cell mobility (10). More invasive metastatic tumors exhibit decreased levels of p63 expression, suggesting that p63 loss upregulates the mechanism of tumorigenesis and metastatic spread $(11,12)$. However, the relationship between GSK-3 $\beta$ and TAp63, p53 family proteins in the migration and invasion of colon cancer cells remains unclear.

The expression of CD74 (major histocompatibility complex class II invariant chain) and CD44 are positively correlated with macrophage migration inhibitory factor (MIF) expression and lymph node metastasis in gastric cancer $(13,14)$. The expression of CD74 significantly increases in gastrointestinal tumors compared to the level of normal tissue (15). Furthermore, stimulation of CD74 in colon cancer cells by MIF enhances cell survival and upregulates Bcl-2 expression (14). 
Engagement of MIF to CD74/CD44 induces upregulation of TAp63 expression, leading to tumor survival and progression (16). However, the role of TAp63 and its association with CD74/CD44 expression in the regulation of cancer metastasis in colon cancer cells remains unclear.

TAp63 also plays an important role in the regulation of energy metabolism: it accumulates in response to metabolic stress and transcriptionally activates NAD-dependent deacetylase sirtuin-1 (SirT1), adenosine monophosphate (AMP)-activated protein kinase (AMPK), and liver kinase B1 (LKB1), resulting in increased fatty acid synthesis (17). Fatty acid synthase (FASN) is selectively expressed in certain human cancers, including breast, stomach and colon cancer. The serum FASN levels of cancer patients in various clinical stages are significantly higher than those of healthy subjects (18). FASN expression is also controlled by p53 family proteins (19). The induction of FASN not only increases tumor growth and invasion but also confers resistance to chemotherapy $(20,21)$. In addition, activation of GSK-3 $\beta$ in murine embryonic fibroblasts induces the phosphorylation of acetyl-CoA carboxylase (ACC), one of the key intracellular lipogenic enzymes (22). The role of GSK-3 $\beta$, and its relationship to TAp63, in fatty acid synthesis for inducing EMT in colon cancer cells also remains unknown.

High levels of TLR4 expression and stimulation are associated with an increased incidence of metastasis and poor prognosis in colorectal cancer (23). Stimulation of TLR4 by lipopolysaccharide (LPS) activates the phosphatidylinositol 3-kinase (PI3K)/AKT (also known as protein kinase B) pathway, leading to an increase in metastasis of colon cancer cells $(24,25)$. PI3K/AKT activation inhibits GSK-3 $\beta$ function by phosphorylation at Ser-9 (26). The LPS-activated GSK-3 $\beta$ signaling pathway is critical for carcinogenesis and metastasis of human cancers $(27,28)$. However, the role of TAp63 in EMT processes and its association with GSK-3 $\beta$ in TLR4-stimulated colorectal cancers (CRCs) remains unclear. Based on previous reports, we investigated the relationship between GSK-3 $\beta$ and TAp63 and the role of these proteins in de novo fatty acid synthesis to regulate invasive activity in LPS-stimulated colon cancer cells. We also examined whether CD74/CD44 expression influences the activity of TAp63 and lipogenesis-mediated EMT of LPS-stimulated CRCs.

\section{Materials and methods}

Cell lines and reagents. The human colorectal cancer cell lines HCT-116, HCT-8 and HT-29 were purchased from the American Type Culture Collection (ATCC; Manassas, VA, USA). These cells were maintained in RPMI-1640 medium (Corning Incorporated, Corning, NY, USA), supplemented with $10 \%$ fetal bovine serum (FBS; RMBIO, Missoula, MT, USA), antibiotics and glutamine at $37^{\circ} \mathrm{C}$ in $5 \% \mathrm{CO}_{2}$. LPS and baicalein were obtained from Sigma-Aldrich (St. Louis, MO, USA). SB216763 was purchased from Selleckchem (Houston, TX, USA).

Western blotting. Cells were washed in phosphate-buffered saline (PBS) and lysed in NP-40 buffer (Elpis Biotech, Inc., Daejeon, Korea) supplemented with a protease inhibitor cocktail (Sigma-Aldrich). Protein phosphorylation states were preserved through the addition of phosphatase inhibitors (Cocktail II; Sigma-Aldrich) to the NP-40 buffer. Protein concentrations were quantified using a BCA assay kit (Pierce, Rockford, IL, USA). Proteins (10 $\mu \mathrm{g} / \mathrm{sample})$ were separated by $10-15 \%$ SDS-PAGE and transferred to nitrocellulose membranes (Millipore Corp., Billerica, MA, USA). The membranes were blocked with 5\% skim milk prior to western blot analysis. Chemiluminescence was detected using an ECL kit (Advansta, Inc., Menlo Park, CA, USA) and the multiple Gel DOC system (Fujifilm, Tokyo, Japan). Primary antibodies against the following proteins were used: E-cadherin, $\mathrm{N}$-cadherin, Snail, vimentin, TCF8/Zeb1, $\beta$-actin, MyD88, LKB1, SirT1, AceCS1, ACSL1, FASN, phospho-ACLY $\left(\operatorname{Ser}^{455}\right)$, ACLY, phospho-ACC $\left(\mathrm{Ser}^{79}\right)$, ACC, phospho-AMPK $\left(\mathrm{Thr}^{172}\right)$, AMPK, phospho-GSK3 $\beta\left(\operatorname{Ser}^{9}\right)$, GSK3 $\beta$, phospho-Lyn $\left(\mathrm{Tyr}^{507}\right)$, Lyn, phospho-PI3K $\left(\mathrm{Tyr}^{428}\right)$, PI3K, phospho-Akt $\left(\mathrm{Ser}^{473}\right)$, Akt, phospho-Src $\left(\mathrm{Tyr}^{416}\right)$ and Src (Cell Signaling Technology, Beverly, MA, USA); TAp63 (BioLegend, Inc., San Diego, CA); $\alpha$-SMA (Bioss, Inc., Woburn, MA, USA); FLAG (SigmaAldrich); CD74, CD44, phospho-PTEN (Ser ${ }^{38 /} / \mathrm{Th}^{382 / 383}$ ), and PTEN (Santa Cruz Biotechnology, Santa Cruz, CA, USA).

Small interfering RNA (siRNA) transfection. Experimentally verified human CD44-small interfering RNA (siRNA) duplex, CD74-siRNA duplex, and negative control-siRNA were obtained from Bioneer (Daejeon, Korea). Cells were seeded at a concentration of $1 \times 10^{5} /$ well in a 6 -well plate and grown overnight prior to transfection with $200 \mathrm{nM}$ siRNA using Lipofectamine RNAiMAX reagent (Invitrogen, Carlsbad, CA, USA) according to the manufacturer's instructions. Cells were used for further experiments $48 \mathrm{~h}$ after transfection.

TAp63 overexpression using transient transfection. Transient transfection of cultured cells was performed using Lipofectamine 2000 according to the manufacturer's instructions. Cells were plated at a density of $2 \times 10^{5} /$ well in a 6-well culture plate and transfected the next day. Typically, $10 \mathrm{ng}$ of construct DNA was transfected with $9 \mu \mathrm{l}$ Lipofectamine. Cultured cells were transiently transfected with either a TAp63 expression vector of TAp63 cDNA cloned into pcDNA3.1 (Addgene, Cambridge, MA, USA) or empty vector pcDNA3.1 (Invitrogen). Cells were transfected for $48 \mathrm{~h}$ and analyzed by western blotting.

Quantification of human cytokines by ELISA. PGE2 and MIF concentrations in the culture supernatants were quantified using a single cytokine ELISA kit from Abcam (Cambridge, UK). VEGF and IL-8 were quantified by a single cytokine ELISA kit from R\&D Systems (Minneapolis, MN, USA) according to the manufacturer's protocol. The data are expressed as the average of the number of biological replicates \pm standard deviation (SD).

Invasion assay. Cell invasion was determined using the CultreCoat 96-well Medium BME Cell Invasion Assay kit (R\&D Systems) according to the manufacturer's protocol. Cells $\left(2.5 \times 10^{4}\right)$ in serum-free RPMI-1640 containing $0.1 \%$ FBS were seeded into the upper chamber and the lower compartment was filled with RPMI-1640 containing 10\% FBS as a chemoattractant. After incubation for $24 \mathrm{~h}$, non-invading 

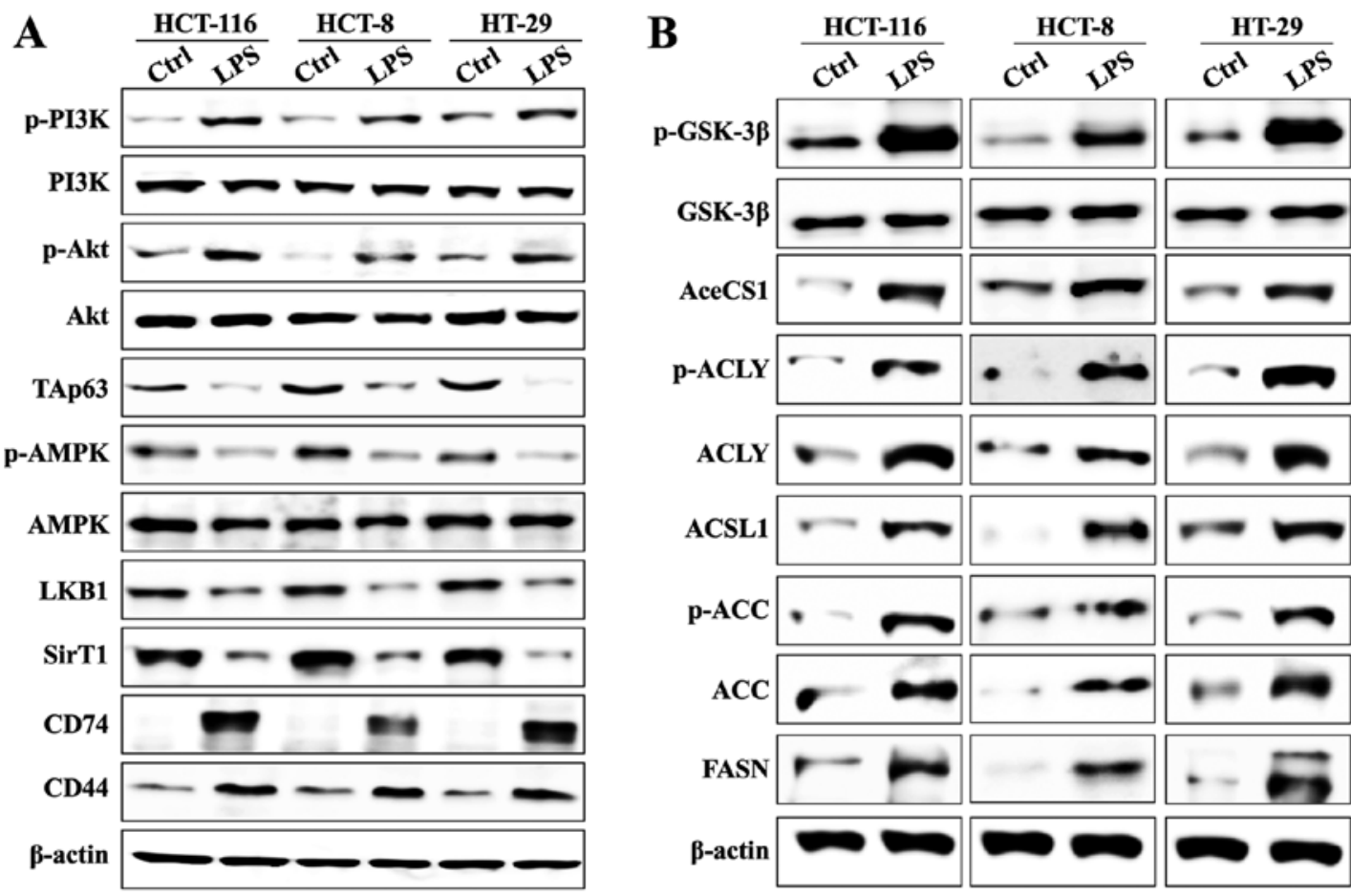

Figure 1. TLR4 stimulation increases the expression of CD74, CD44 and lipogenic enzymes in colon cancer cells. HCT-116, HCT-8 and HT-29 cells were treated with $500 \mathrm{ng} / \mathrm{ml}$ LPS for $24 \mathrm{~h}$. Sterile distilled water was used as a vehicle control. Total cell lysates were subjected to western blot analysis with

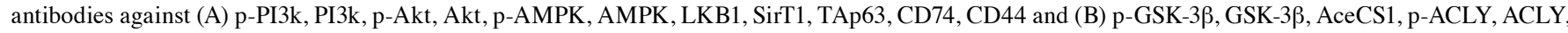
ACSL1, p-ACC, ACC or FASN. $\beta$-actin served as an internal control. Data are representative of 3 independent experiments.

cells on the upper membrane surface were removed by wiping with a cotton swab. Invaded cells were stained with calceinAM and quantified using a microplate reader.

Measurement of free fatty acid level. For assessment of free fatty acids, colorectal cancer cells were harvested, homogenized with chloroform/Triton X-100 solution (1\% Triton X-100 in pure chloroform), and centrifuged at top speed to collect the organic phase (lower phase). The supernatant was vacuum dried to remove the chloroform and the dried lipid samples were dissolved in fatty acid assay buffer. Concentrations of free fatty acids were determined using the Free Fatty Acid Quantification kit (Abcam) according to the manufacturer's instructions.

Statistical analysis. Data were expressed as the mean \pm standard deviation (SD). Statistical analysis was conducted using one-way analysis of the variance. A P-value $<0.05$ was considered statistically significant.

\section{Results}

TLR4 stimulation increases the expression of GSK-3 , CD74, CD44 and lipogenic enzymes in colon cancer cells. First, we investigated whether TLR4 stimulation affects the expression of TAp63-related signal molecules and the expression of lipogenic enzymes in CRC cells. LPS-treated colon cancer cells (HCT-116, HCT-8 and HT-29) showed activation of the PI3K/AKT signaling pathway, whereas phosphorylation of AMPK, LKB1 and SirT1, key metabolic regulators, was significantly reduced (Fig. 1A). Although the expression of TAp63 was downregulated, upregulation of CD74 and CD44 was observed in LPS-activated HCT-116, HCT-8 and HT-29 cells (Fig. 1A). Enzymes associated with lipogenesis in CRC were activated after engagement of LPS with TLR4. Cytoplasmic acetyl-CoA synthetase (AceCS1), ATP-citrate lyase (ACLY), phospho-ACLY (p-ACLY), long-chain acylCoA synthetase (ACSL1), ACC, phospho-ACC (p-ACC), and fatty acid synthase (FASN) were all upregulated. In addition, LPS activation induced phosphorylation of GSK-3 $\beta$ in colon cancer cells (Fig. 1B). Our results suggest that the TLR4mediated signaling pathway is one of the key regulators of metastasis-related mechanisms in colon cancer cells.

Inhibition of GSK-3 $\beta$ restores the expression of TAp63 and attenuates fatty acid synthesis, secretion of MIF and generation of PGE2 in LPS-activated colon cancer cells. To confirm the correlation of GSK-3 $\beta$ with lipogenic enzyme activity and invasion of LPS-activated colon cancer cells, the cells were pretreated with the GSK-3 $\beta$ inhibitor SB216763. Treatment with SB216763 blocked phosphorylation of GSK-3 $\beta$ and attenuated activation of lipogenic enzymes in LPS-treated colon cancer cells (Fig. 2A). The levels of intracellular long chain fatty acid increased $>3$-fold compared to non-treated control cells after stimulation with LPS. In addition, pretreatment with SB216763 significantly blocked fatty acid synthesis in LPS-activated colon cancer cells (Fig. 2B). Prostaglandin $\mathrm{E}_{2}$ (PGE2) increases the phosphorylation of GSK-3 $\beta$ and consequently stimulates $\beta$-catenin/T cell factor (TCF)-mediated transcription for growth and invasion in colon cancer (29). We observed that stimulation with LPS increased the secretion of PGE2 in colon cancer cells; however, inhibition of GSK-3 $\beta$ 

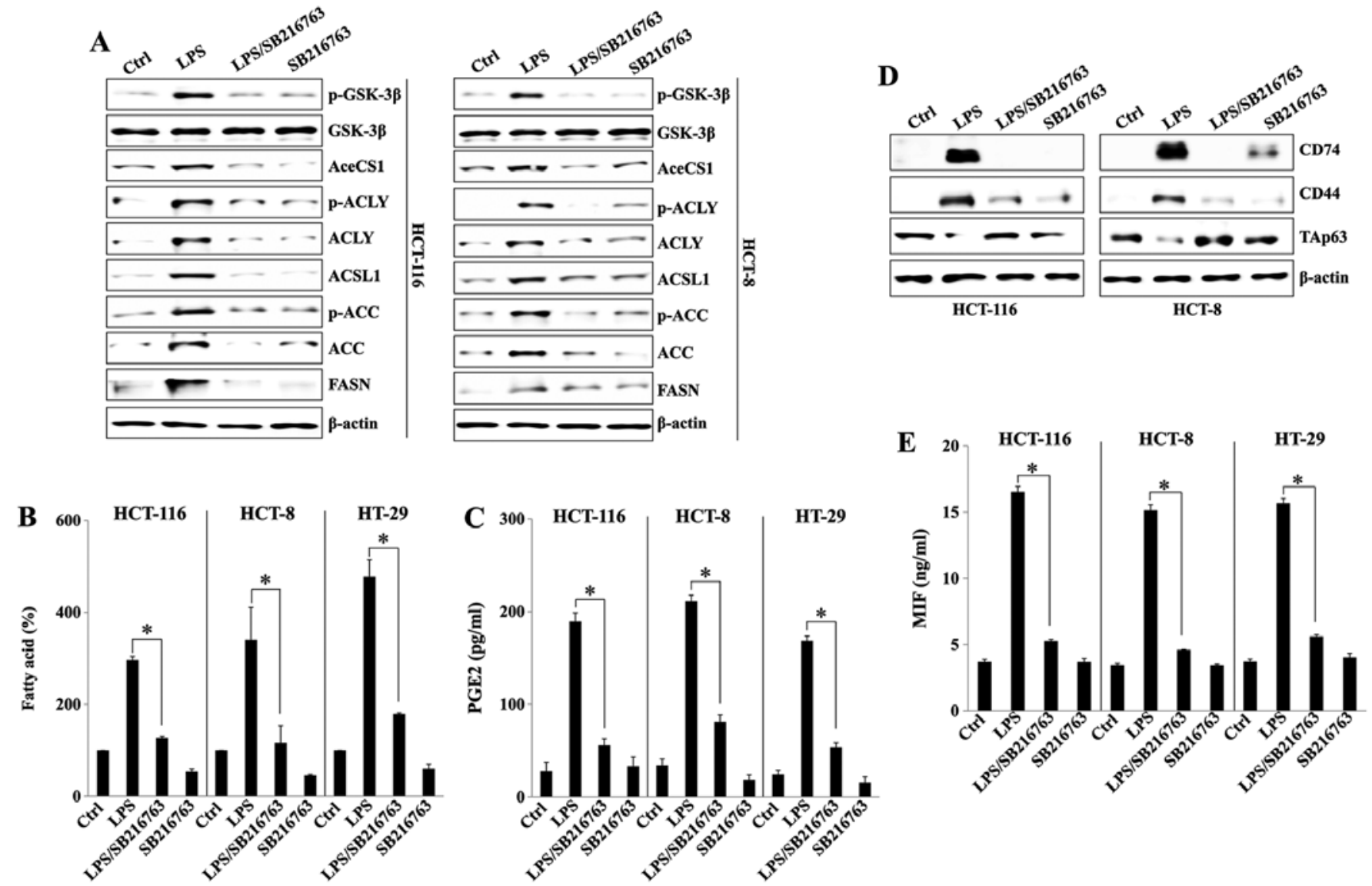

Figure 2. Inhibition of GSK-3 $\beta$ attenuates fatty acid synthesis and secretion of MIF. The effect of GSK-3 $\beta$ inhibitor on intracellular lipogenesis enzymes, fatty acid synthesis and PGE2 production was investigated. (A) Total protein was subjected to western blotting with antibodies against (A) p-GSK-3 $\beta$, GSK-3 $\beta$, AceCS1, p-ACLY, ACLY, ACSL1, p-ACC, ACC and FASN. (B) Production of intracellular long chain fatty acid levels in LPS-stimulated colon cancer cells compared to non-treated control cells was determined using a free fatty acid quantification kit. Data from at least three independent experiments performed in triplicate are represented as percentages of individual values in $\mathrm{nmol} / \mu \mathrm{l}$ compared to control values (set at $100 \%$ ) and are shown as means $\pm \mathrm{SD}$. ${ }^{*} \mathrm{P}<0.05$. (C) Concentrations of PGE2 in culture supernatants of HCT-116, HCT-8 and HT-29 cells were quantified by ELISA. Cells were seeded into 6-well plates $\left(2 \times 10^{5} /\right.$ well) and treated with $500 \mathrm{ng} / \mathrm{ml} \mathrm{LPS}$ for $24 \mathrm{~h}$. ${ }^{*} \mathrm{P}<0.01$. Data are presented as the mean of three independent experiments and error bars represent SDs of the means. (D) Total protein was subjected to western blot analysis with antibodies against TAp63, CD44 and CD74. (E) Concentrations of MIF in the culture supernatants of HCT-116, HCT-8 and HT-29 cells were quantified by ELISA. Cells were seeded into 6-well plates (2x10 $/$ well) and treated with $500 \mathrm{ng} / \mathrm{ml}$ LPS for $24 \mathrm{~h} .{ }^{*} \mathrm{P}<0.01$. Data are presented as the mean of three independent experiments and error bars represent the SD of the mean.

reduced the LPS-activated PGE2 production (Fig. 2C). Treatment with SB216763 effectively blocked the expression of CD74 and CD44 in LPS-treated colon cancer cells (Fig. 2D). SB216763 also reduced secretion of MIF in LPS-treated colon cancer cells (Fig. 2E). In addition, suppression of GSK-3 $\beta$ recovered the expression of TAp63 in LPS-stimulated colon cancer cells (Fig. 2D). Our results suggest that GSK-3 $\beta$ not only plays a critical role in lipogenesis and cancer metabolism, but also regulates the level of TAp63 in LPS-stimulated colon cancer cells.

TAp63 expression regulates GSK-3 $\beta$-dependent intracellular lipogenesis and invasion activity of LPS-treated colon cancer cells. Next, we investigated the role and connection of TAp63 in GSK-3 $\beta$-mediated lipogenesis and EMT processes in CRCs after LPS stimulation. After transfection with the TAp63expressing plasmid, the levels of TAp63-related proteins did not significantly change compared to control cells or cells transfected with a control vector (Fig. 3A). TAp63 overexpression in LPS-activated HCT-116 cells not only blocked the attenuation of phosphorylated AMPK, LKB1 and SirT1, but also prevented the upregulation of phosphorylated GSK-3 $\beta$ and activation of lipogenic enzymes (Fig. 3B and C). Although, the upregulated CD74 and CD44 expression following LPS stimulation was maintained (Fig. 3B), the downregulation of lipogenic enzymes was accompanied by decreased intracellular fatty acid synthesis (Fig. 3D). Inhibition of GSK-3 $\beta$ by TAp63 transfection also reduced the production of PGE2 (Fig. 3D). TAp63-expressing HCT-116 cells activated by LPS showed concomitant reduction of the mesenchymal markers $\mathrm{N}$-cadherin, snail, vimentin, $\alpha$-SMA and TCF8 and decreased migratory activity (Fig. 3E and F). Our results suggest that TAp63 expression regulates GSK-3 $\beta$-related intracellular lipogenesis and influences mesenchymal characteristics and invasion activity in LPS-activated CRCs.

TLR4-triggered CD74/CD44 induction contributes to lipogenesis-dependent EMT processes of colon cancer cells through the regulation of TAp63 expression. We found that treatment with SB216763 recovered the expression of TAp63 in LPS-activated colon cancer cells, whereas overexpression of TAp63 also reduced the levels of phosphorylated GSK-3 $\beta$. 


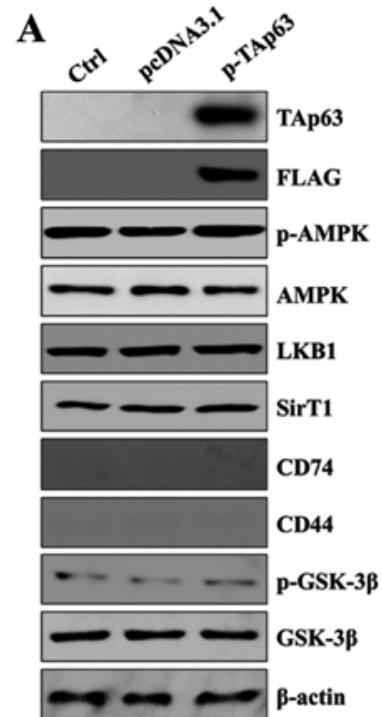

B

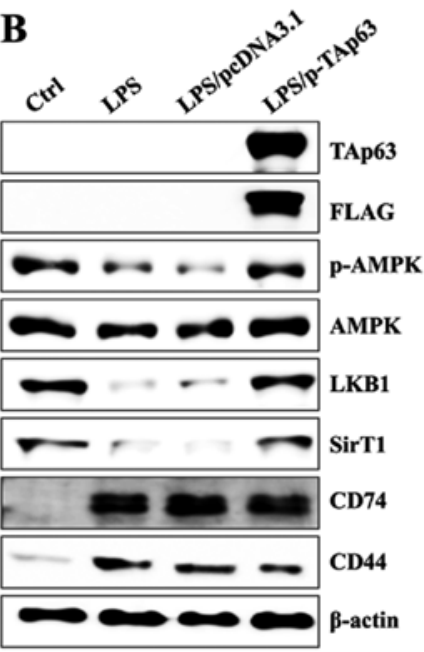

C
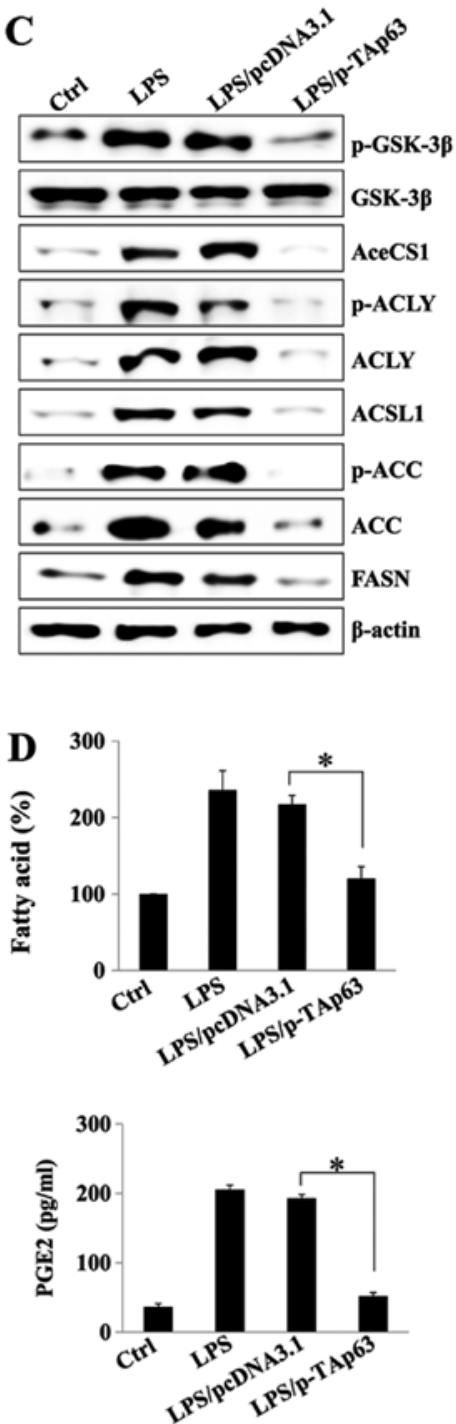

$\mathbf{E}$

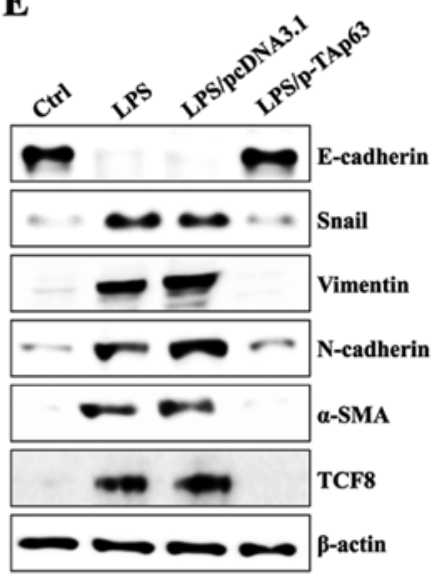

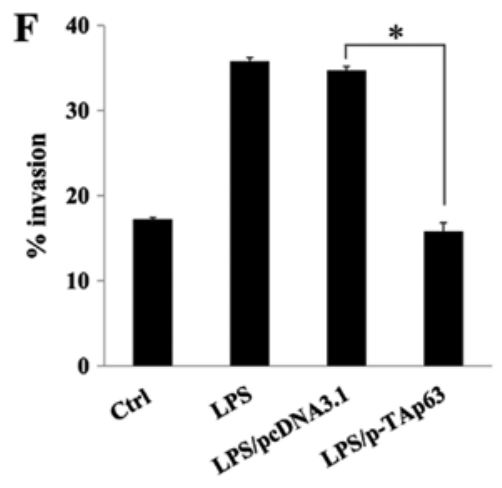

Figure 3. TAp63 expression regulates GSK-3 $\beta$-dependent intracellular lipogenesis and invasion activity of LPS-treated colon cancer cells. (A) The HCT-116 cells were transfected with either empty vector pcDNA3.1 or TAp63 expression vector. The cells were analyzed by western blotting with anti-TAp63 and anti-FLAG antibodies and then total protein was subjected to immunoblotting with antibodies against CD74, CD44, p-AMPK, AMPK, LKB1, SirT1, GSK-3 $\beta$ and p-GSK-3 3 . HCT-116 cells were treated with $500 \mathrm{ng} / \mathrm{ml} \mathrm{LPS} \mathrm{for} 24 \mathrm{~h}$ and then transfected with either empty vector pcDNA3.1 or TAp63 expression vector. (B and C) Total proteins in cell lysates were subjected to western blot analysis of TAp63, FLAG, CD74, CD44, p-AMPK, AMPK, LKB1, or SirT1 (B), and p-GSK-3 $\beta$, GSK-3 $\beta$, AceCS1, p-ACLY, ACLY, ACSL1, p-ACC, ACC or FASN (C). $\beta$-actin served as an internal control. (D) Production of intracellular long chain fatty acid levels in LPS-stimulated colon cancer cells was determined using a free fatty acid quantification kit and compared to that of non-treated control cells. Data from at least three independent experiments performed in triplicate are represented as percentage of individual values in nmol $/ \mu 1 \mathrm{compared}$ to control values (set at 100\%) and are shown as means $\pm \mathrm{SD}$. ${ }^{*} \mathrm{P}<0.01$. Concentration of PGE2 in the culture supernatants of HCT-116 cells was quantified by ELISA. Cells were seeded into 6 -well plates $\left(2 \times 10^{5} /\right.$ well) and treated with $500 \mathrm{ng} / \mathrm{ml} \mathrm{LPS} \mathrm{for} 24 \mathrm{~h}$. ${ }^{*} \mathrm{P}<0.01$. Data are presented as the mean of three independent experiments and error bars represent SDs of the means. (E) Western blot analysis of EMT markers E-cadherin, N-cadherin, vimentin, Snail, $\alpha$-SMA and TCF8/Zeb1. $\beta$-actin served as an internal control. (F) TAp63 overexpression decreased cell invasiveness detected by a BME cell invasion assay kit. Each value is the mean \pm standard deviation of 3 determinations. ${ }^{*} \mathrm{P}<0.01$.

We estimated that another molecule plays an important role in regulating TAp63 expression and GSK-3ß-mediated lipogenesis. From previous results, targeted inhibition of GSK-3 $\beta$ using SB216763 suppressed the expression of CD74 and CD44, but reversed the level of TAp63. Moreover, TAp63 overexpression and reduction of lipogenesis had no effect on the expression of CD74 and CD44 in LPS-treated colon cancer cells. Based on these results, we next evaluated whether LPS-induced expression of CD74 and CD44 affects the expression of TAp63 and phosphorylated GSK-3 $\beta$, the activation of lipogenic enzymes, fatty acid synthesis and MIF secretion. Although TLR4-stimulation of CD44 or CD74-knockdown HCT-116 cells induced PI3K/AKT activation, gene silencing of CD44 or CD74 with siRNA blocked the downregulation of TAp63, phosphorylated AMPK, LKB1 and SirT1 in LPS-treated HCT-116 cells (Fig. 4A and B). Expression of FASN and the production of long chain free fatty acids in LPS-activated HCT-116 cells were decreased after knockdown of CD44 or CD74 with siRNA (Fig. 4A-C). Secretion of PGE2 and MIF was also attenuated in CD44 or CD74 knockdown HCT-116 cells after stimulation with LPS (Fig. 4D and E). Expression of mesenchymal markers and invasion activity were significantly suppressed when induction of CD44 or CD74 expression was inhibited in LPS-stimulated HCT-116 cells (Fig. 5A 

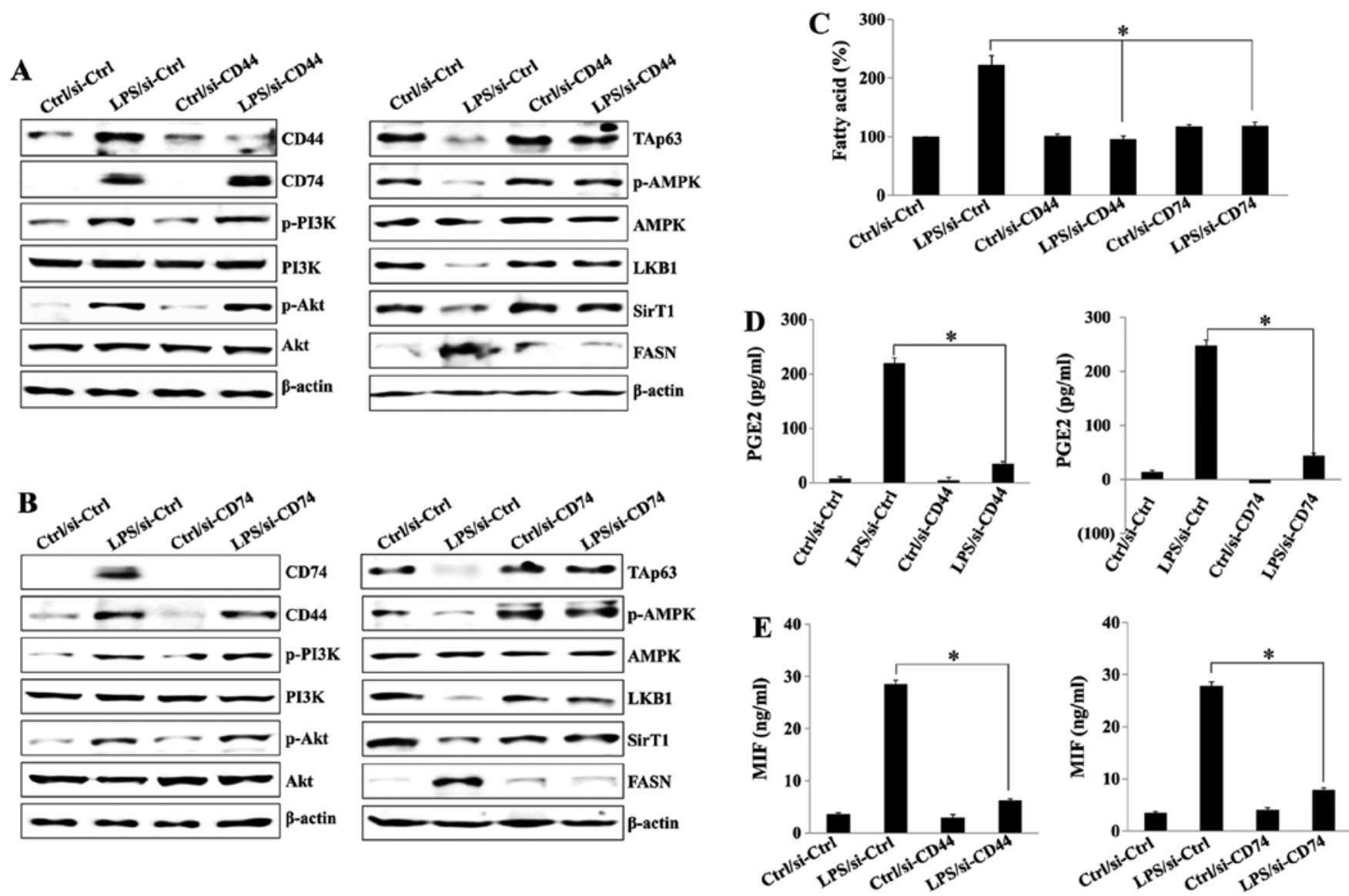

Figure 4. Induction of CD74 and CD44 by TLR4 stimulation contributes to fatty acid synthesis through PI3K/Akt activation. HCT-116 cells were treated with $500 \mathrm{ng} / \mathrm{ml} \mathrm{LPS} \mathrm{for} 24 \mathrm{~h}$ and then transfected with CD44-siRNA $(200 \mathrm{nM})$, CD74-siRNA $(200 \mathrm{nM})$, or control-siRNA for $48 \mathrm{~h}$ prior to experiments. (A and B) Total protein was subjected to western blot analysis with antibodies against CD44, CD74, p-PI3k, PI3k, p-Akt, Akt, TAp63, p-AMPK, AMPK, LKB1, SirT1 or FASN. $\beta$-actin served as an internal control. (C) Production of intracellular long chain fatty acids in LPS-stimulated colon cancer cells was determined using the free fatty acid quantification kit and compared to that of non-treated control cells. Data from at least three independent experiments performed in triplicate are represented as percentage of individual values in $\mathrm{nmol} / \mu \mathrm{l}$ compared to control values (set at $100 \%$ ) and are shown as means $\pm \mathrm{SD}$. ${ }^{*} \mathrm{P}<0.01$. (D and E) Concentrations of PGE2 (D) and MIF (E) in culture supernatants of HCT-116 cells were quantified by ELISA. Cells were seeded onto 6 -well plates $\left(2 \times 10^{5} /\right.$ well $)$ and treated with $500 \mathrm{ng} / \mathrm{ml}$ LPS for $24 \mathrm{~h} .{ }^{*} \mathrm{P}<0.01$. Data are presented as the mean of three independent experiments and error bars represent the SD of the mean.

and B). The production of EMT-related cytokines, including VEGF and IL-8, after LPS stimulation was also suppressed in HCT-116 cells transfected with CD44 or CD74 siRNA (Fig. 5C). Our results suggest that the CD74/CD44 expression of TLR4-stimulated colon cancer cells plays a critical role in the suppression of TAp63 as well as lipogenesis-related EMT processes.

Baicalein prevents migratory activity of LPS-treated colon cancer cells via modulation of TAp63/GSK-3 $\beta$-mediated cancer metabolism. Baicalein, a natural plant extract, suppresses EMT of breast epithelial cells and the tumorigenic activity of breast cancer cells (30). We previously reported that treatment with baicalein upregulates TAp63 expression, resulting in apoptosis of B-cell lymphoma cell lines (31). Treatment with baicalein also decreased the lipid level in the livers of mice fed a high-fat diet through PI3K/AKT-mediated AMPK activation (32). Based on these results, we investigated whether baicalein prevents the lipogenesis-related EMT processes through the regulation of TAp63-dependent GSK-3 $\beta$ expression. Pre-exposure of LPS-activated HCT-116 cells to baicalein showed reduced phosphorylation of Syk/Src tyrosine kinase, resulting in blockage of PI3K/AKT activation (Fig. 6A). Treatment with baicalein not only prevented the downregulation of TAp63 and phosphorylated AMPK, LKB1, and SirT1, but also suppressed the expression of CD44, CD74, and phosphorylated GSK-3 $\beta$ in LPS-treated HCT-116 cells (Fig. 6B). The production of long chain fatty acid and PGE2 in TLR4-activated HCT-116 cells was efficiently blocked after treatment with baicalein (Fig. 6C and D). Expression of mesenchymal markers (Fig. 6E), production of MIF (Fig. 6F), and migratory activity (Fig. 6G) of LPS-stimulated HCT-116 cells were also inhibited by baicalein. Our results suggest that baicalein controls the EMT processes of LPS-stimulated CRCs through the regulation of TAp63/GSK-3 $\beta$-mediated lipogenesis.

\section{Discussion}

Epithelial to mesenchymal transition (EMT), a process in which epithelial cell layers lose their polarity through dramatic remodeling of the cytoskeleton, plays an important role in tumor metastasis (33). TLR4 expressed in several types of tumor cells plays a vital role in carcinogenesis, metastasis and 

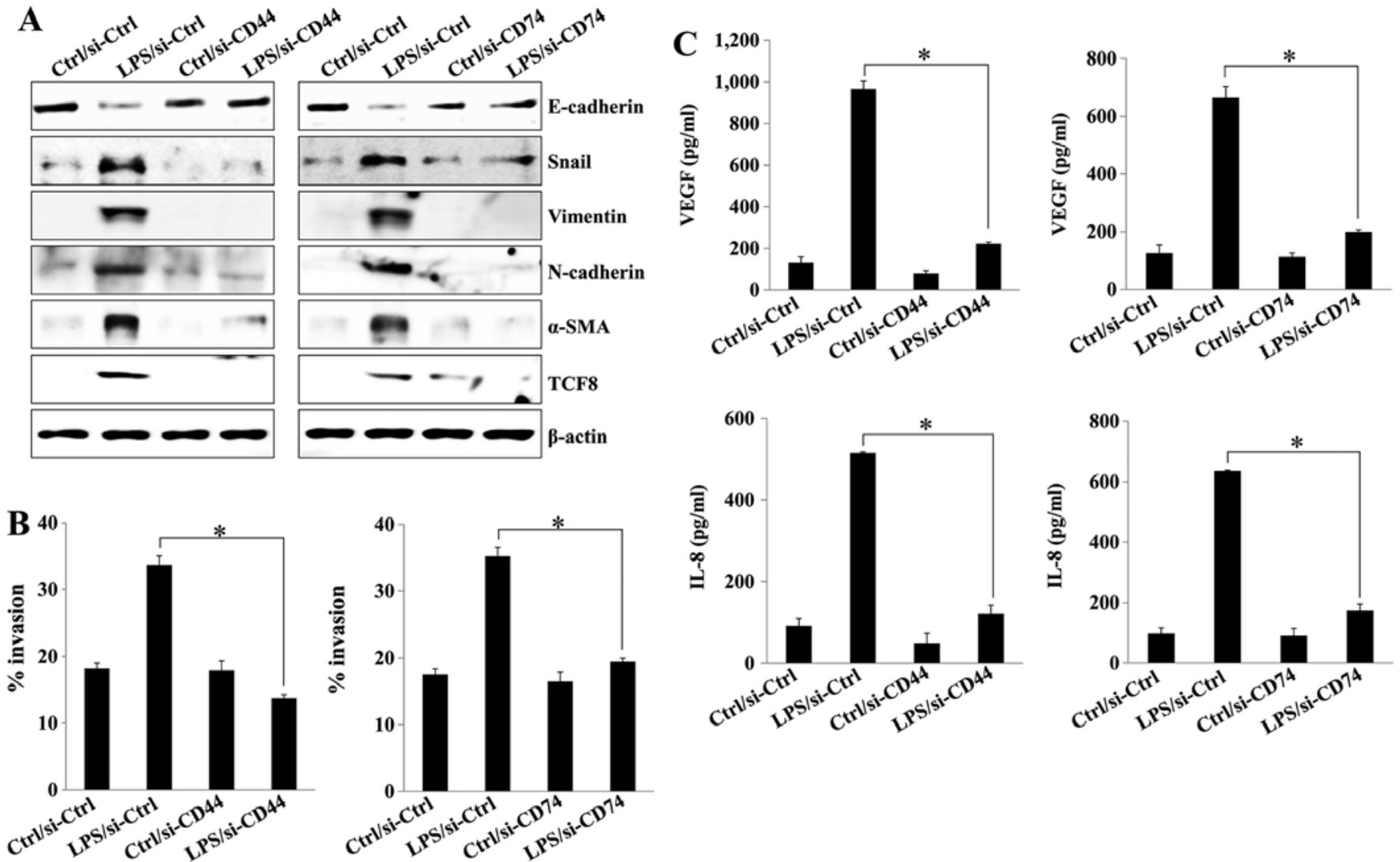

Figure 5. Induction of CD74 and CD44 by TLR4 stimulation contributes to EMT and invasiveness. HCT-116 cells were seeded into 6-well plates (2x105/well), treated with $500 \mathrm{ng} / \mathrm{ml}$ LPS for $24 \mathrm{~h}$, and then transfected with CD44-siRNA (200 nM), CD74-siRNA (200 nM), or control-siRNA for $48 \mathrm{~h}$ prior to experiments. (A) Western blot analysis of EMT markers (E-cadherin, N-cadherin, vimentin, Snail, $\alpha$-SMA and TCF8/Zeb1). $\beta$-actin served as an internal control. (B) Gene silencing of CD44 or CD74 with siRNA transfection decreased the invasiveness of TLR4-stimulated HCT-116 cells. Migratory activity was detected using a BME cell invasion assay kit as described in Materials and methods. Each value is the mean \pm standard deviation of three determinations. $\mathrm{P}<0.05$. (C) Concentrations of VEGF and IL-8 in culture supernatants of CD44- or CD74-knockdown HCT-116 cells were quantified by ELISA. "P<0.05. Data are presented as the mean of three independent experiments and error bars represent the SD of the mean.

cancer progression (34). LPS enhances the invasive potential and induces EMT in HCC cells in a TLR4-dependent manner through activation of nuclear factor kappa B (NF-kB) (28). Expression of p63 has been detected in several cancers, including bladder (35) and lung (36). Disruption of p63 in squamous cell lines results in upregulation of genes associated with increased invasiveness and metastasis in tumors (35). These results are consistent with the role of p63 as a marker of non-invasive epithelial tumors (37). Levels of p63 have wide-ranging influences on biological processes, including metabolism. TAp63 overexpressing cells are more glycolytically active than $\triangle \mathrm{Np} 63$ cells, indicating that the two isoforms may regulate key steps of glycolysis in an opposite manner (38). However, the detailed regulatory mechanisms of TAp63 in colon cancer metastasis and its association with GSK-3 $\beta$-mediated intracellular lipogenesis remain obscure. We observed that TLR4-stimulated CRCs showed suppressed expression of TAp63, but upregulated levels of phosphorylated GSK-3 $\beta$ and lipogenic enzymes. The migratory capacity and expression of EMT-related markers were also increased in a GSK-3 $\beta$-dependent manner after TLR4 activation. Overexpression of TAp63 significantly blocked GSK-3 $\beta$ phosphorylation and fatty acid synthesis. Pharmacological inhibition of GSK-3 $\beta$ also prevented lipogenesis. Our results suggest that TLR4 activation in colon cancer cells enhances EMT-related intracellular lipid metabolism and migratory activity through modulation of TAp63-dependent GSK-3 $\beta$ expression (Fig. 7).

FASN is highly expressed in many types of human cancer, including colorectal cancer (39). FASN levels in the serum of colorectal cancer patients are significantly higher than those of healthy individuals. Serum FASN levels in colorectal cancer patients are associated with tumor extent, liver metastasis and tumor clinical stage (39-41). Nuclear accumulation of GSK-3 $\beta$ is also significantly correlated with poor prognosis, distant metastasis, and loss of membranous $\beta$-catenin colon cancer (42). However, the relationship between lipogenesis and GSK-3 $\beta$ in TLR4-stimulated colorectal cancer remains unclear. The LKB1/AMPK signaling pathway regulates energy homeostasis through its ability to balance catabolic and anabolic activity to regulate lipid and glucose metabolism (43). Phosphorylation of the lipogenic enzyme ACC by AMPK is critical for the production of malonyl-CoA as a substrate for fatty acid biosynthesis. Expression of TAp63 is required for transcriptional activation of AMPK, LKB1 and SirT1 (17). TLR4 stimulation of CRCs reduced TAp63 expression as well as activation of AMPK, LKB1 and SirT1. In addition, we showed that upregulation of phosphorylated GSK-3 $\beta$ by 

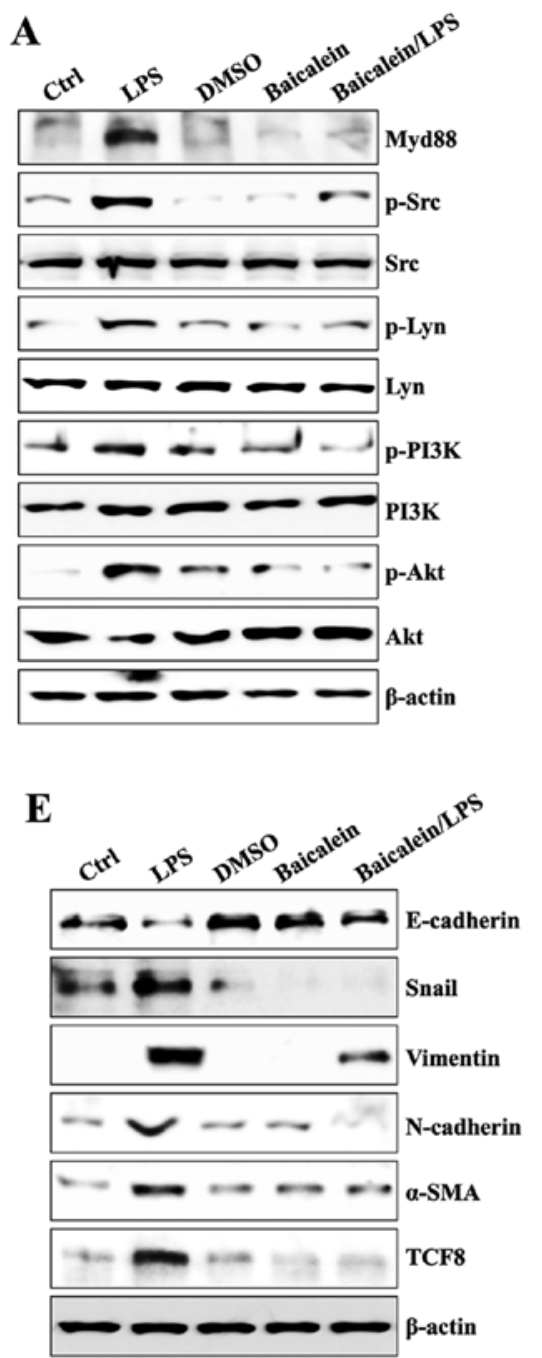
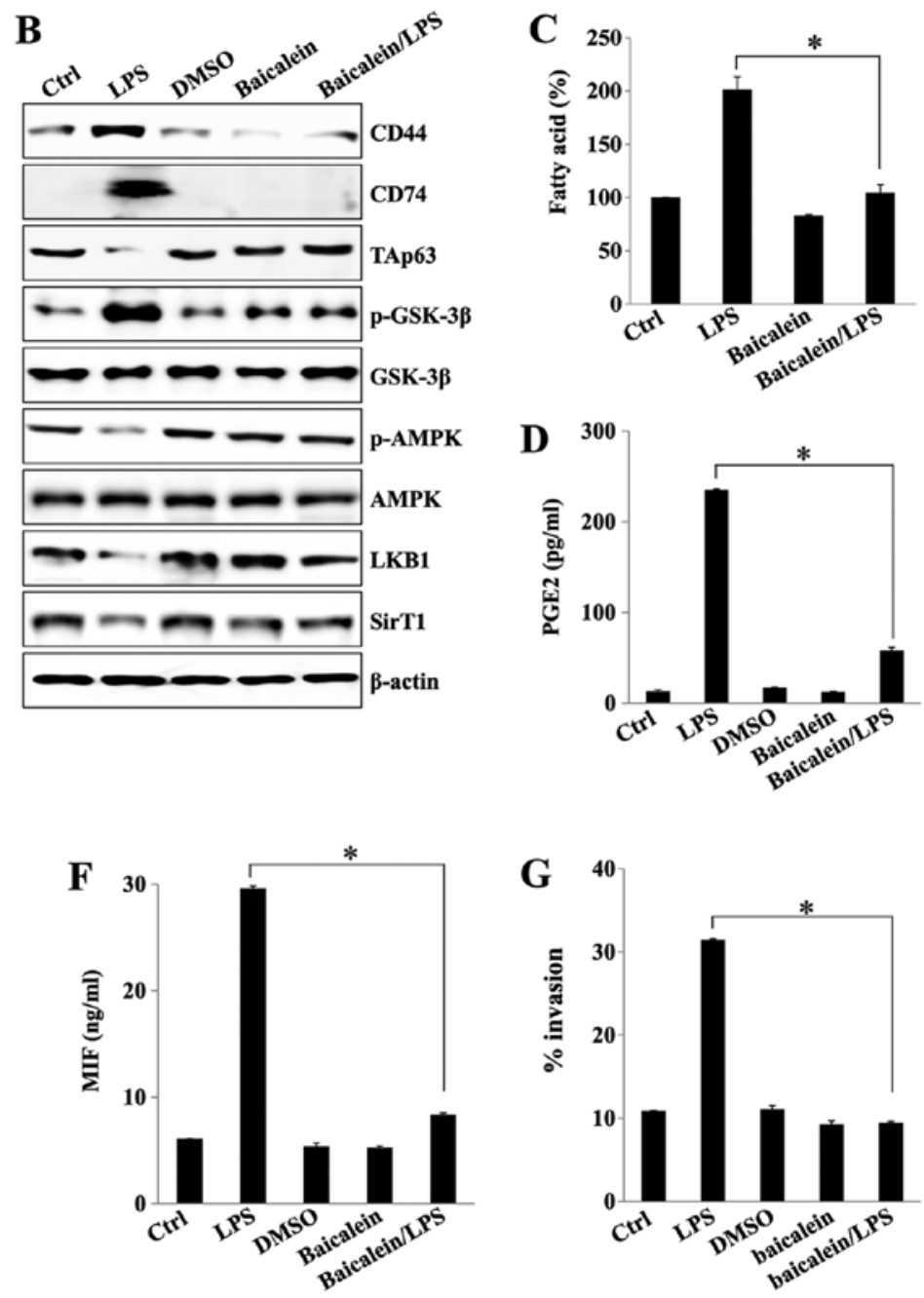

Figure 6. Baicalein inhibits migratory activity of LPS-treated colon cancer cells by modulation of GSK-3 $\beta$. HCT-116 cells were preincubated with $20 \mu \mathrm{M}$ baicalein for $2 \mathrm{~h}$ and then treated with $500 \mathrm{ng} / \mathrm{ml}$ LPS for $24 \mathrm{~h}$. (A, B and E) Total cell lysates were subjected to western blotting with antibodies against (A)

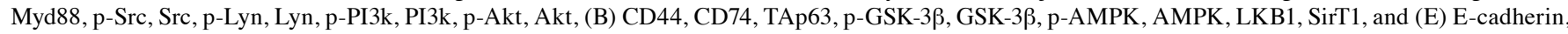
$\mathrm{N}$-cadherin, vimentin, Snail, $\alpha$-SMA or TCF8/Zeb1. $\beta$-actin served as an internal control. (C) Production of intracellular long chain fatty acids in LPSstimulated colon cancer cells was determined using a free fatty acid quantification kit and compared to that of non-treated control cells. Data from at least three independent experiments performed in triplicate are represented as a percentage of individual values in $\mathrm{nmol} / \mu \mathrm{l}$ compared to control values (set at $100 \%$ ) and are shown as mean \pm SD. " $\mathrm{P}<0.05$. (D and F) Concentrations of PGE2 (D) and MIF (F) in culture supernatants of HCT-116 cells were quantified by ELISA. Cells were seeded onto 6-well plates ( $2 \times 10^{5} /$ well) and treated with $500 \mathrm{ng} / \mathrm{ml}$ LPS for $24 \mathrm{~h}$. ${ }^{*} \mathrm{P}<0.005$. Data are presented as the mean of three independent experiments and error bars represent the SD of the mean. (G) Baicalein inhibited invasiveness following TLR stimulation, as detected by a BME cell invasion assay kit as described in Materials and methods. ${ }^{*} \mathrm{P}<0.005$. Each value is the mean \pm standard deviation of 3 determinations.

LPS treatment triggered the stimulation of intracellular lipogenic enzymes, fatty acid synthesis and invasion activity of CRCs. Moreover, TAp63 overexpression suppressed GSK-3 $\beta$ mediated lipogenesis and cancer metastasis in LPS-activated colon cancer cells. These results suggest that TLR4-mediated TAp63 expression is associated with GSK-3 $\beta$ phosphorylation and lipogenesis-related cancer progression.

CD74 is usually expressed at high levels by antigenpresenting cells (APCs), including B cells, monocytes, macrophages and dendritic cells in normal tissues (44). The TLR4 agonist LPS, a bacterial cell wall component, regulates CD44 expression in human monocytes (45). More recently, CD74 expression on epithelial cells has been shown to be related to chronic inflammation and carcinogenesis in the gastrointestinal tract (46). We observed that the levels of TAp63 and phosphorylated GSK-3 $\beta$ of LPS-stimulated colon cancer cells were expressed in an opposite manner. As expected, targeted inhibition of GSK-3 $\beta$ with SB216763 prevented the induction of CD74, CD44 and MIF expression in LPS-activated CRCs. Moreover, overexpression of TAp63 failed to inhibit CD74/CD44 expression, but significantly suppressed intracellular lipogenesis. Based on these results, we investigated the association between CD74/CD44 and TAp63 as well as the role of CD74/CD44 on TAp63/GSK-3ß-mediated lipogenesis. Although gene silencing of CD44 or CD74 with siRNA reduced the expression of FASN and fatty acid production in addition to blocking invasion activity, downregulation of CD74 and CD44 failed to reduce the expression of TAp63 and related metabolic regulators, including AMPK, LKB1 and SirT1, in LPS-stimulated HCT-116 cells. These results suggest that GSK-3 3-dependent CD74/CD44 expression plays a role in downregulation of TAp63 expression and indirectly controls 


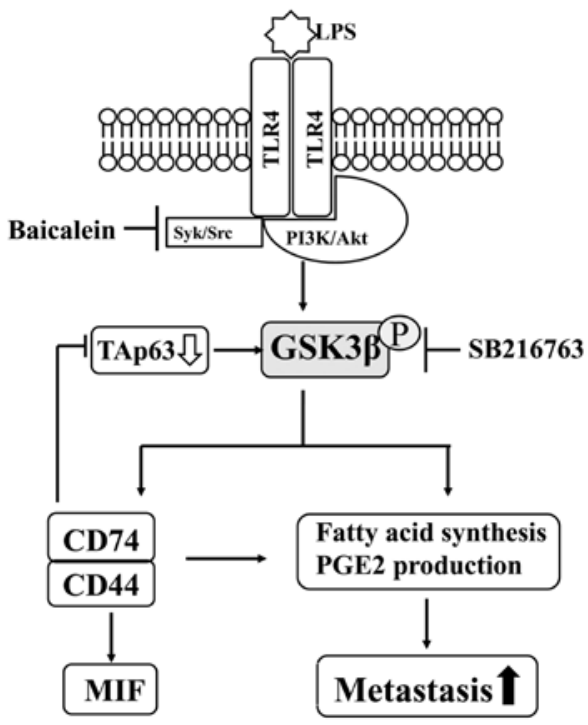

Figure 7. Schematic diagram of intracellular signaling mechanisms during LPS-induced TLR4 activation in human colorectal cancer cells. Interaction of LPS with TLR4 on colorectal cancer cells (HCT-116, HCT-8 and HT-29) transduces cellular signals through a common pathway that leads to activation of Syk/Src and PI3K/Akt. Subsequent downregulation of TAp63 by stimulation of TLR4 expression results in phosphorylation of GSK-3 $\beta$, upregulation of the MIF/CD74/CD44 signaling pathway, increased fatty acid synthesis, and PGE2 production, leading to metastasis of colon cancer cells. Treatment of colon cancer cells with baicalein rescues the induction of metastasis and GSK-3 $\beta$-mediated fatty acid synthesis by LPS-stimulated TLR activation. Our findings suggest that metastasis of colon cancer cells may be caused by decreased TAp63 expression and increased phosphorylation of GSK-3 $\beta$, upregulation of CD74/CD44 and production of PGE2 and MIF.

GSK-3 $\beta$-mediated intracellular lipid synthesis and EMT processes of LPS-activated colon cancer cells (Fig. 7).

Treatment of B-celllymphoma cells with baicalein increases the expression of TAp63 to induce apoptosis and concomitantly reduces the levels of CD74 and CD44 (31). Baicalein treatment of LPS-stimulated HCT-116 cells also reversed the level of TAp63 and expression of related metabolic regulators. We also observed that baicalein reduced the activity of GSK-3 $\beta$-dependent lipogenic enzymes, CD74/CD44 expression, and fatty acid-related migratory activity in LPS-treated CRCs. These results suggest baicalein as a new candidate therapeutic drug for the control of lipogenesis-related colon cancer metastasis. TLR4 agonist, LPS derived from intestinal microflora, influences the growth of cancer cells (47). Conventional chemotherapy against colon cancer enhances the expression of TLR4 (48). Our results suggest that inhibition of the expression of CD44 or blocking CD44-mediated signaling pathway is critical for the conservation of TAp63 after treatment with anticancer drug. Based on these results, targeting inhibition of CD44 using specific agent or preventing the activation of CD44 using a newly developed drug, such as baicalein is important to control the metastasis of colon cancer in vivo animal model. Further studies are needed to examine these possibilities.

In conclusion, the present study demonstrates that the TAp63/GSK-3 $\beta$ signaling pathway might be a critical regulator of lipogenesis-related cancer metastasis and that regulation of CD74/CD44 expression in colon cancer cells could be a promising target for TAp63-mediated cancer metastasis.

\section{Acknowledgements}

This study was supported by the Basic Science Research Program of the Ministry of Education (2015R1D1A1A01056672) and the Ministry of Science, ICT \& Future Planning (NRF-2015R1C1A2A01053732) through the National Research Foundation of Korea (NRF) of the Republic of Korea.

\section{References}

1. Amar S, Belmaker RH and Agam G: The possible involvement of glycogen synthase kinase-3 (GSK-3) in diabetes, cancer and central nervous system diseases. Curr Pharm Des 17: 2264-2277, 2011.

2. Qu L, Huang S, Baltzis D, Rivas-Estilla AM, Pluquet O, Hatzoglou M, Koumenis C, Taya Y, Yoshimura A and Koromilas AE: Endoplasmic reticulum stress induces p53 cytoplasmic localization and prevents p53-dependent apoptosis by a pathway involving glycogen synthase kinase-3beta. Genes Dev 18: 261-277, 2004.

3. Charvet $\mathrm{C}$ and Maurer U: GSK-3 turns p53 deadly. Cell Cycle 10: 3621-3622, 2011.

4. Kim NH, Kim HS, Li XY, Lee I, Choi HS, Kang SE, Cha SY, Ryu JK, Yoon D, Fearon ER, et al: A p53/miRNA-34 axis regulates Snaill-dependent cancer cell epithelial-mesenchymal transition. J Cell Biol 195: 417-433, 2011.

5. Cha YH, Kim NH, Park C, Lee I, Kim HS and Yook JI: MiRNA-34 intrinsically links p53 tumor suppressor and Wnt signaling. Cell Cycle 11: 1273-1281, 2012.

6. Sadot E, Geiger B, Oren M and Ben-Ze'ev A: Down-regulation of beta-catenin by activated p53. Mol Cell Biol 21: 6768-6781, 2001.

7. Wong AS and Gumbiner BM: Adhesion-independent mechanism for suppression of tumor cell invasion by E-cadherin. J Cell Biol 161: 1191-1203, 2003.

8. Yang A and McKeon F: P63 and P73: P53 mimics, menaces and more. Nat Rev Mol Cell Biol 1: 199-207, 2000.

9. Gressner O, Schilling T, Lorenz K, Schulze Schleithoff E, Koch A, Schulze-Bergkamen H, Lena AM, Candi E, Terrinoni A, Catani MV, et al: TAp63alpha induces apoptosis by activating signaling via death receptors and mitochondria. EMBO J 24: 2458-2471, 2005.

10. Yang A, Kaghad M, Caput D and McKeon F: On the shoulders of giants: p63, p73 and the rise of p53. Trends Genet 18: 90-95, 2002.

11. Urist MJ, Di Como CJ, Lu ML, Charytonowicz E, Verbel D, Crum CP, Ince TA, McKeon FD and Cordon-Cardo C: Loss of p63 expression is associated with tumor progression in bladder cancer. Am J Pathol 161: 1199-1206, 2002.

12. Koga F, Kawakami S, Fujii Y, Saito K, Ohtsuka Y, Iwai A, Ando N, Takizawa T, Kageyama Y and Kihara K: Impaired p63 expression associates with poor prognosis and uroplakin III expression in invasive urothelial carcinoma of the bladder. Clin Cancer Res 9: 5501-5507, 2003.

13. Zheng YX, Yang M, Rong TT, Yuan XL, Ma YH, Wang ZH, Shen LS and Cui L: CD74 and macrophage migration inhibitory factor as therapeutic targets in gastric cancer. World $\mathrm{J}$ Gastroenterol 18: 2253-2261, 2012.

14. Maharshak N, Cohen S, Lantner F, Hart G, Leng L, Bucala R and Shachar I: CD74 is a survival receptor on colon epithelial cells. World J Gastroenterol 16: 3258-3266, 2010.

15. Gold DV, Stein R, Burton J and Goldenberg DM: Enhanced expression of CD74 in gastrointestinal cancers and benign tissues. Int J Clin Exp Pathol 4: 1-12, 2010.

16. Shachar I and Haran M: The secret second life of an innocent chaperone: The story of CD74 and B cell/chronic lymphocytic leukemia cell survival. Leuk Lymphoma 52: 1446-1454, 2011.

17. Su X, Gi YJ, Chakravarti D, Chan IL, Zhang A, Xia X, Tsai KY and Flores ER: TAp63 is a master transcriptional regulator of lipid and glucose metabolism. Cell Metab 16: 511-525, 2012.

18. Notarnicola M, Tutino V, Calvani M, Lorusso D, Guerra V and Caruso MG: Serum levels of fatty acid synthase in colorectal cancer patients are associated with tumor stage. J Gastrointest Cancer 43: 508-511, 2012.

19. D'Erchia AM, Tullo A, Lefkimmiatis K, Saccone C and Sbisà E: The fatty acid synthase gene is a conserved p53 family target from worm to human. Cell Cycle 5: 750-758, 2006. 
20. Visca P, Sebastiani V, Pizer ES, Botti C, De Carli P, Filippi S, Monaco S and Alo PL: Immunohistochemical expression and prognostic significance of FAS and GLUT1 in bladder carcinoma. Anticancer Res 23 (1A): 335-339, 2003.

21. Alò PL, Visca P, Botti C, Galati GM, Sebastiani V, Andreano T, Di Tondo U and Pizer ES: Immunohistochemical expression of human erythrocyte glucose transporter and fatty acid synthase in infiltrating breast carcinomas and adjacent typical/atypical hyperplastic or normal breast tissue. Am J Clin Pathol 116: 129-134, 2001.

22. Terrand J, Bruban V, Zhou L, Gong W, El Asmar Z, May P, Zurhove K, Haffner P, Philippe C, Woldt E, et al: LRP1 controls intracellular cholesterol storage and fatty acid synthesis through modulation of Wnt signaling. J Biol Chem 284: 381-388, 2009.

23. Cammarota R, Bertolini V, Pennesi G, Bucci EO, Gottardi O, Garlanda C, Laghi L, Barberis MC, Sessa F, Noonan DM, et al: The tumor microenvironment of colorectal cancer: Stromal TLR-4 expression as a potential prognostic marker. J Transl Med 8: 112,2010

24. Doan HQ, Bowen KA, Jackson LA and Evers BM: Toll-like receptor 4 activation increases Akt phosphorylation in colon cancer cells. Anticancer Res 29: 2473-2478, 2009.

25. Hsu RY, Chan CH, Spicer JD, Rousseau MC, Giannias B, Rousseau S and Ferri LE: LPS-induced TLR4 signaling in human colorectal cancer cells increases betal integrin-mediated cell adhesion and liver metastasis. Cancer Res 71: 1989-1998, 2011.

26. Gong R, Rifai A and Dworkin LD: Activation of PI3K-AktGSK3beta pathway mediates hepatocyte growth factor inhibition of RANTES expression in renal tubular epithelial cells. Biochem Biophys Res Commun 330: 27-33, 2005.

27. Earl TM, Nicoud IB, Pierce JM, Wright JP, Majoras NE, Rubin JE, Pierre KP, Gorden DL and Chari RS: Silencing of TLR4 decreases liver tumor burden in a murine model of colorectal metastasis and hepatic steatosis. Ann Surg Oncol 16: 1043-1050, 2009.

28. Jing YY, Han ZP, Sun K, Zhang SS, Hou J, Liu Y, Li R, Gao L, Zhao X, Zhao QD, et al: Toll-like receptor 4 signaling promotes epithelial-mesenchymal transition in human hepatocellular carcinoma induced by lipopolysaccharide. BMC Med 10: 98, 2012.

29. Shao J, Jung C, Liu C and Sheng H: Prostaglandin E2 Stimulates the beta-catenin/T cell factor-dependent transcription in colon cancer. J Biol Chem 280: 26565-26572, 2005.

30. Chung H, Choi HS, Seo EK, Kang DH and Oh ES: Baicalin and baicalein inhibit transforming growth factor- $\beta 1$-mediated epithelial-mesenchymal transition in human breast epithelial cells. Biochem Biophys Res Commun 458: 707-713, 2015.

31. Park GB, Kim YS, Lee HK, Yang JW, Kim D and Hur DY: ASK1/ JNK-mediated TAp63 activation controls the cell survival signal of baicalein-treated EBV-transformed B cells. Mol Cell Biochem 412: 247-258, 2016.

32. Pu P, Wang XA, Salim M, Zhu LH, Wang L, Chen KJ, Xiao JF, Deng W, Shi HW, Jiang H, et al: Baicalein, a natural product, selectively activating AMPK $\alpha_{2}$ and ameliorates metabolic disorder in diet-induced mice. Mol Cell Endocrinol 362: 128-138, 2012.

33. Jing Y,Han Z, Zhang S, Liu Y and Wei L: Epithelial-mesenchymal transition in tumor microenvironment. Cell Biosci 1: 29, 2011.

34. Rakoff-Nahoum S and Medzhitov R: Toll-like receptors and cancer. Nat Rev Cancer 9: 57-63, 2009.
35. Barbieri CE, Tang LJ, Brown KA and Pietenpol JA: Loss of p63 leads to increased cell migration and up-regulation of genes involved in invasion and metastasis. Cancer Res 66: 7589-7597, 2006.

36. Shimada Y, Ishii G, Nagai K, Atsumi N, Fujii S, Yamada A, Yamane Y, Hishida T, Nishimura M, Yoshida J, et al: Expression of podoplanin, CD44, and p63 in squamous cell carcinoma of the lung. Cancer Sci 100: 2054-2059, 2009.

37. Wang TY, Chen BF, Yang YC, Chen H, Wang Y, Cviko A, Quade BJ, Sun D, Yang A, McKeon FD, et al: Histologic and immunophenotypic classification of cervical carcinomas by expression of the p53 homologue p63: A study of 250 cases. Hum Pathol 32: 479-486, 2001.

38. D'Aguanno S, Barcaroli D, Rossi C, Zucchelli M, Ciavardelli D, Cortese C, De Cola A, Volpe S, D'Agostino D, Todaro M, et al: p63 isoforms regulate metabolism of cancer stem cells. J Proteome Res 13: 2120-2136, 2014.

39. Zaytseva YY, Rychahou PG, Gulhati P, Elliott VA, Mustain WC, O'Connor K, Morris AJ, Sunkara M, Weiss HL, Lee EY, et al: Inhibition of fatty acid synthase attenuates CD44-associated signaling and reduces metastasis in colorectal cancer. Cancer Res 72: 1504-1517, 2012.

40. Long QQ, Yi YX, Qiu J, Xu CJ and Huang PL: Fatty acid synthase (FASN) levels in serum of colorectal cancer patients: Correlation with clinical outcomes. Tumour Biol 35: 3855-3859, 2014.

41. Murata S, Yanagisawa K, Fukunaga K, Oda T, Kobayashi A, Sasaki R and Ohkohchi N: Fatty acid synthase inhibitor cerulenin suppresses liver metastasis of colon cancer in mice. Cancer Sci 101: 1861-1865, 2010.

42. Salim T, Sjölander A and Sand-Dejmek J: Nuclear expression of glycogen synthase kinase- $3 \beta$ and lack of membranous $\beta$-catenin is correlated with poor survival in colon cancer. Int J Cancer 133: 807-815, 2013.

43. Towler MC and Hardie DG: AMP-activated protein kinase in metabolic control and insulin signaling. Circ Res 100: 328-341, 2007.

44. Roche PA, Teletski CL, Stang E, Bakke O and Long EO: Cell surface HLA-DR-invariant chain complexes are targeted to endosomes by rapid internalization. Proc Natl Acad Sci USA 90: 8581-8585, 1993

45. Gee K, Lim W, Ma W, Nandan D, Diaz-Mitoma F, Kozlowski M and Kumar A: Differential regulation of CD44 expression by lipopolysaccharide (LPS) and TNF-alpha in human monocytic cells: Distinct involvement of c-Jun N-terminal kinase in LPS-induced CD44 expression. J Immunol 169: 5660-5672, 2002.

46. Borghese F and Clanchy FI: CD74: An emerging opportunity as a therapeutic target in cancer and autoimmune disease. Expert Opin Ther Targets 15: 237-251, 2011

47. Santaolalla R, Sussman DA, Ruiz JR, Davies JM, Pastorini C, España CL, Sotolongo J, Burlingame O, Bejarano PA, Philip S, et al: TLR4 activates the $\beta$-catenin pathway to cause intestinal neoplasia. PLoS One 8: e63298, 2013.

48. Chung YH and Kim D: Enhanced TLR4 expression on colon cancer cells after chemotherapy promotes cell survival and epithelial-mesenchymal transition through phosphorylation of GSK-3 $\beta$. Anticancer Res 36: 3383-3394, 2016. 